\title{
Impact of Light Intensity on Sleep of Patients in the Intensive Care Unit: A Prospective Observational Study
}

\author{
Piotr F Czempik ${ }^{1}$, Agnieszka Jarosińska², Krystyna Machlowska ${ }^{3}$, Michał Pluta ${ }^{4}$
}

\begin{abstract}
Aims and objectives: Sleep deprivation in the intensive care unit (ICU) has been linked to numerous complications. Light levels might impact the sleep of patients in the ICU. The aim of the study was to measure light levels during sleep-protected time in the ICU and to assess the impact of light intensity on sleep quantity/quality.

Materials and methods: This prospective, observational study was conducted in a 10-bed, mixed surgical/medical ICU. For measuring light levels, a commercially available smartphone application was used. The measurements were performed between 23:30 and 06:15 hours at 15-minute intervals. To assess sleep quantity, we used Patient's Sleep Observation Behavioral Tool and to assess sleep quality, we used Richards-Campbell Sleep Scale. Results: The median number of time points at which patients were asleep was 20 (interquartile range, IQR 14-23) out of 25 (5 hours). The median self-reported quality of sleep (overall score) was 49 (IQR 28-71). The median values for individual questions are: question 1 (sleep depth) - 54.0 (IQR 37-78), question 2 (sleep latency) - 40.5 (IQR 6-90), question 3 (awakenings) - 52.5 (IQR 28-76), question 4 (returning to sleep)—25.5 (IQR $11-78)$, and question 5 (sleep quality)-67.5 (IQR 5-76). No correlation was found between self-reported sleep quality and time spent asleep $(p=0.36)$. There was no correlation between average light levels during sleep-protected time and sleep quantity $(p=0.42) / \mathrm{sleep}$ quality $(p=$ 0.13). There was a correlation between average ( $13 \pm 5$ lux) light levels before sleep-protected time and sleep quality $(p=0.008)$.

Conclusion: Mean light levels of $11 \pm 9$ lux during sleep-protected time have no negative impact on quantity and quality of sleep in intensive care unit patients. Light levels up to 18 lux directly before falling asleep improve patients' self-reported quality of sleep in the ICU.

Clinical significance: Finding safe levels of light intensity during sleep-protected time in ICU.

Keywords: Critical care, Delirium, Intensive care unit, Light, Sleep quality.

Indian Journal of Critical Care Medicine (2020): 10.5005/jp-journals-10071-23323
\end{abstract}

\section{INTRODUCTION}

Sleep disruption is common in patients hospitalized in the ICU.? Polysomnography studies in ventilated and nonventilated patients show that sleep is characterized by severe fragmentation, frequent awakenings, increased light sleep, and decreased rapid eye movement sleep, with approximately half of total sleep time occurring during daytime. ${ }^{2}$ Sleep deprivation in the intensive has been linked to delirium, neurocognitive dysfunction, prolonged mechanical ventilation, impaired immune function, impaired wound healing, and delayed recovery from critical illness. ${ }^{1,3,4}$ Sleep deprivation is a source of anxiety during hospitalization in the ICU. ${ }^{5}$ Intensive care unit-related factors that influence sleep quality are light, noise (alarms, respirators, pagers, telephones, and conversations $)^{6}$, patient care activities, diagnostic procedures, and medications commonly used in the ICU. ${ }^{7}$

Intensive care unit is a unique location in a hospital where highly specialized care is provided for critically ill patients 24 hours a day. The nature of a disease, intensive treatment, and nursing around the clock lead to circadian rhythm disruption. ${ }^{8-10}$ Disrupted circadian rhythms are associated with impaired survival from critical illness. ${ }^{11}$ Circadian rhythm disturbances, similarly to sleep disruption, lead to delirium. ${ }^{12}$ An element of circadian rhythm disruption is change in the usual day/night pattern of light exposure when a patient is cared for in the ICU. Patients are exposed to 24-hour nursing care with unnaturally low light levels during the day and constant light interruptions through the night. ${ }^{13}$ Light is the most important factor that influences circadian rhythm. In a healthy person, numerous biological processes are under circadian control: optimal sleep patterns, rapid healing, recovery from a disease, to name just a few. ${ }^{14}$
${ }^{1}$ Department of Anaesthesiology and Intensive Care, Faculty of Medical Sciences in Katowice, Medical University of Silesia, Katowice, Poland

${ }^{2-4}$ Students' Scientific Society, Department of Anaesthesiology and Intensive Care, Faculty of Medical Sciences in Katowice, Medical University of Silesia, Katowice, Poland

Corresponding Author: Piotr F Czempik, Department of Anaesthesiology and Intensive Care, Faculty of Medical Sciences in Katowice, Medical University of Silesia, Katowice, Poland, Phone: +48 505087875, e-mail: pczempik@sum.edu.pl

How to cite this article: Czempik PF, Jarosińska A, Machlowska K, Pluta M. Impact of Light Intensity on Sleep of Patients in the Intensive Care Unit: A Prospective Observational Study. Indian J Crit Care Med 2020;24(1):33-37.

Source of support: Nil

Conflict of interest: None

Interventions have been postulated to reduce lighting intensity in the ICU during nocturnal hours. ${ }^{15} \mathrm{~A}$ longitudinal study in critical care implemented nonpharmacological environmental changes to reduce night disruption and demonstrated an impressive reduction in delirium and an improvement in sleep. The interventions tested in the study included noise reduction, light reduction, and specific patient care interventions. ${ }^{16}$ Current ICU guidelines recommend natural daylight for every patient room and artificial light that can be dialed up and down; currently, there is no indication as to what daytime light intensity should be achieved. ${ }^{17}$

Although there are numerous factors associated with sleep deprivation in the ICU, light intensity is a factor that can be modified. The link between this environmental factor and sleep 
quantity/quality in the ICU is still under investigation. Therefore, the aim of our study was to measure light levels during sleepprotected time in ICU and assess the impact of light intensity on sleep quantity/quality during sleep-protected time in the ICU.

\section{Materials and Methods}

\section{Study Design and Setting}

This was a prospective, observational study. The study was conducted in the ICU of a university-affiliated tertiary care center. The ICU is a mixed surgical/medical unit with 10 intensive care beds: 8 beds in a multi-bed room (bays separated by curtains, a nursing station located approximately in the middle of the room), 2 beds in single-bed rooms.

Artificial lighting consists of overhead panels and spotlights at every bedside. The overhead lighting is usually switched off during the sleep-protected time, whereas the lights at the nursing station are dimmed. The window blinds are kept open during the day and closed during the night (sleep-protected time). As this was an observational study, the local bioethics committee decided that no ethics committee approval was required (KNW/0022/KB/56/18). All patients gave their informed consent for participation in the study and appropriate consent forms were signed. The observation period spanned over a year, from March 2018 to April 2019. The observations were performed during single night in patients who remained in an ICU for at least 24 hours, and we have not recorded if this was first or following night for a particular patient. The ICU personnel was aware of the concept of the study.

\section{Study Population}

We aimed to recruit 30 patients. We used the following eligibility criteria: neurological status allowing communication with a patient and ICU length of stay of at least 24 hours. We assessed both spontaneously breathing and mechanically ventilated patients (mode of ventilation was not recorded). We did not use any exclusion criteria, not even preexisting sleep disorders for that matter. We collected basic demographic and clinical data: age; sex; illness severity according to 3 classification systems Acute Physiology and Chronic Health Evaluation II (APACHE II), Simplified Acute Physiology Score II (SAPS II) and Sequential Organ Failure Assessment (SOFA). To monitor the level of sedation, the Richmond agitation-sedation scale (RASS) was used. ${ }^{18}$ We used a protocol of minimal sedation (i.e., RASS -2 to 0 ) in order for patients to be comfortable, calm, and cooperative. ${ }^{19}$ Pain intensity was monitored using modified Numerical Rating Scale (mNRS). We aimed at 0-1 points in all patients at all times. We screened patients for delirium using Polish version of Confusion Assessment Method for ICU (CAM-ICU). ${ }^{20}$

\section{Light Levels Measurements}

For measuring light levels, we used a commercially available smartphone application. As we did not find any applications that were tested against standard methods of measuring light intensity in our setting, we decided to use a freely available smartphone application (LUX Light Meter, Nipakul Buttua) written for iOS operating system (Apple Inc., United States of America). The light levels were measured using the smartphone's front camera (light sensor) directed upward, located approximately $50 \mathrm{~cm}$ above a patient's head. Great care was given to not to occlude the light sensor in any way, keeping it clean at all times, in order to obtain the most accurate results. The measurements were performed between 23:30 and 06:15 hours at 15-minute intervals for all study subjects in the manner described above, which corresponded to 25 time points during sleep-protected time (00:00-06:00 hours) and 3 time points outside sleep-protected time (23:30, 23:45, 06:15 hours). The measurements were performed by one of the researchers (AJ, KM) using a single mobile phone dedicated for the purposes of the study.

\section{Sleep Quantity and Quality Assessment}

To assess sleep quantity, we used Patient's Sleep Observation Behavioral Tool. This tool involves observation of sleep/wakefulness in 15-minute intervals. This tool has been used to assess sleep and sleep-promoting interventions in critically ill patients. ${ }^{21}$ When a patient was awake at any time during sleep-protected time, we assessed pain using mNRS and additional doses of painkillers were offered to patients when required. If a patient was awake, he/she was also screened for delirium using CAM-ICU, and if positive, an attending physician would be notified. Following sleep-protected time, patients were asked to complete sleep quality satisfaction questionnaire-Richards-Campbell Sleep Scale (RCSS). ${ }^{22}$ The patients completed sleep quality questionnaires themselves and if mobility issues precluded ability to write, one of the researchers (AJ, KM) was of assistance.

\section{Statistical Analysis}

Statistical analysis was carried out using licensed MedCalc Statistical Software version 18 (MedCalc Software bvba, Ostend, Belgium; https://www.medcalc.org; 2018) by one of the researchers (MP). Quantitative variables with normal distribution were presented as an arithmetic mean and standard deviation ( \pm SD). In case of nonnormal distribution, the variables were represented as a median and IQR. The character of the distribution of quantitative variables was verified by the Shapiro-Wilk test. The differences in quantitative variables were assessed using the analysis of variance or the Kruskal-Wallis test. A Chi-square test or Fisher's exact test was used for the qualitative variables (in the case of a small subgroup size). Correlations were evaluated using Pearson's linear correlation coefficient or Spearman's rank. The assumed statistical significance was $p<0.05$.

\section{Results}

The study population characteristics are presented in Table 1. There were 25 time points during designated sleep-protected time at which we assessed whether patients were asleep or awake. The median number of time points at which patients were asleep was 20 (IQR 14-23) out of 25, so the median time asleep for the study group was 5 (IQR 3.5-5.7) hours. There were no inter-sex differences as far as quantity $(p=0.7)$ and quality $(p=0.8)$ of sleep were concerned.

Table 1: Study population characteristics

\begin{tabular}{ll}
\hline Characteristic & Value \\
\hline Sex ratio: men/women $n(\%)$ & $18(58) / 13(42)$ \\
Age (mean \pm SD) (years) & $54 \pm 14$ \\
APACHE II (mean \pm SD) (points) & $11 \pm 6$ \\
SAPS II (mean \pm SD) (points) & $32 \pm 10$ \\
SOFA (mean \pm SD) (points) & $6 \pm 4$ \\
Mechanically ventilated patients $n(\%)$ & $4(13)$ \\
Patients hospitalized in single-bed room $n(\%)$ & $8(26)$ \\
\hline
\end{tabular}

APACHE, acute physiology and chronic health evaluation; SAPS II, simplified acute physiology score II; SD, standard deviation; and SOFA, sequential organ failure assessment 
The median self-reported quality of sleep (overall score) was 49 (IQR 28-71) out of $100 \mathrm{~mm}$. The median values for individual questions are: question 1 (sleep depth) - 54.0 (IQR 37-78) mm, question 2 (sleep latency) - 40.5 (IQR 6-90) mm, question 3 (awakenings) -52.5 (IQR 28-76) $\mathrm{mm}$, question 4 (returning to sleep) -25.5 (IQR 11-78) mm, and question 5 (sleep quality) - 67.5 (IQR 5-76) $\mathrm{mm}$.

There was no correlation between severity of disease as per APACHE II $(p=0.90)$, SAPS II $(p=0.88)$, and SOFA $(p=0.77)$ and self-reported quality of sleep.

The number of time points at which patients were reporting pain during sleep-protected time was 7 (NRS 1: 5 times, NRS 2: 1 time, NRS 3: 1 time). No patient reported pain sensation before sleep-protected time (i.e., 23:30-23:45). There was also no correlation between the number of time points at which patients reported pain sensation and sleep quality $(p=0.34)$.

There were $2(7 \%)$ patients experiencing delirium. There was no correlation between incidence of delirium and sleep quantity/ quality.

No correlation was found between self-reported sleep quality and time spent asleep ( $p=0.36$ ).

We compared light levels directly before (23:30-23:45 hours) and during sleep-protected time (00:00-06:00 hours) (Fig. 1).

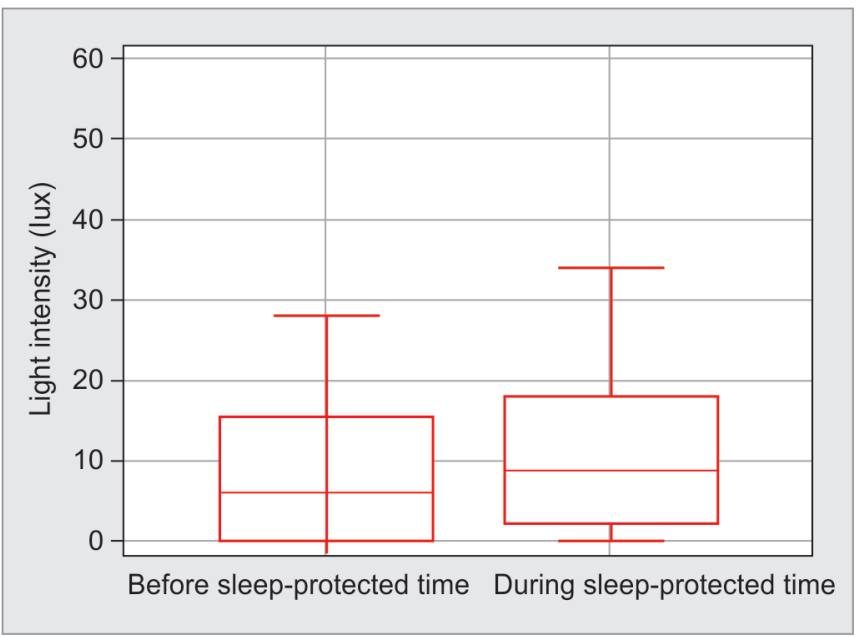

Fig. 1: Light intensity directly before and during sleep-protected time
Median light levels before and during sleep-protected time were 6 (IQR 0-16) and 9 (IQR 2-18) lux, respectively.

Light levels at 15-minute intervals during sleep-protected time are presented in Figure 2.

The light levels started to rise at approximately 05:15 hours, although this was still inside the sleep-protected time.

There was no correlation between average light levels during sleep-protected time (11 \pm 9 lux) and sleep quantity $(p=0.42)$ / sleep quality $(p=0.13)$.

We found a correlation between sleep quality above average light levels before sleep-protected time ( $13 \pm 5$ lux $)$ and improved sleep quality $(p=0.008)$. The discussed correlations are summarized in Table 2.

\section{Discussion}

In our study, we aimed at assessing impact of light intensity on sleep quantity and quality of patients hospitalized in the ICU. When starting on such a project one has to decide how to measure sleep quantity/quality. There are several methods at our disposal that can be broadly divided into objective and subjective. Polysomnography is a gold standard. Actigraphy is another standard objective method. Nevertheless, these objective methods are difficult to use in the ICU. ${ }^{8}$ Observational tools are semiobjective, but are very simple to use in the ICU. ${ }^{23}$ We intended to perform a practical study, that does not require special equipment, so we decided to use an observational tool and a patient questionnaire to assess sleep of patients in our ICU. On the other hand, there are numerous subjective methods that we can use: RCSS, Verran Snyder Harper Sleep Scale, Pittsburgh Sleep Quality Index, Insomnia Severity Index, and Sleep Efficiency Index. ${ }^{24}$ In our study, we decided to use one of the available observation tools (Patient's Sleep Observation Behavioral Tool) and one of the available patient questionnaires (RCSS). RichardsCampbell Sleep Scale is a 5-item visual analog scale widely used in ICUs that has good reliability and validity. ${ }^{25}$ Although there may be some discrepancies between observer reported and patient's self-reported sleep quality using this instrument, ${ }^{26,27}$ it is the most practical tool to be used in the ICU.

In our study, we analyzed sleep quantity/quality of patients during 6-hour sleep-protected time. Although most adults sleep approximately 7.5 hours a day, it is rarely achievable in the ICU due to intensive treatment and nursing care. The median self-reported

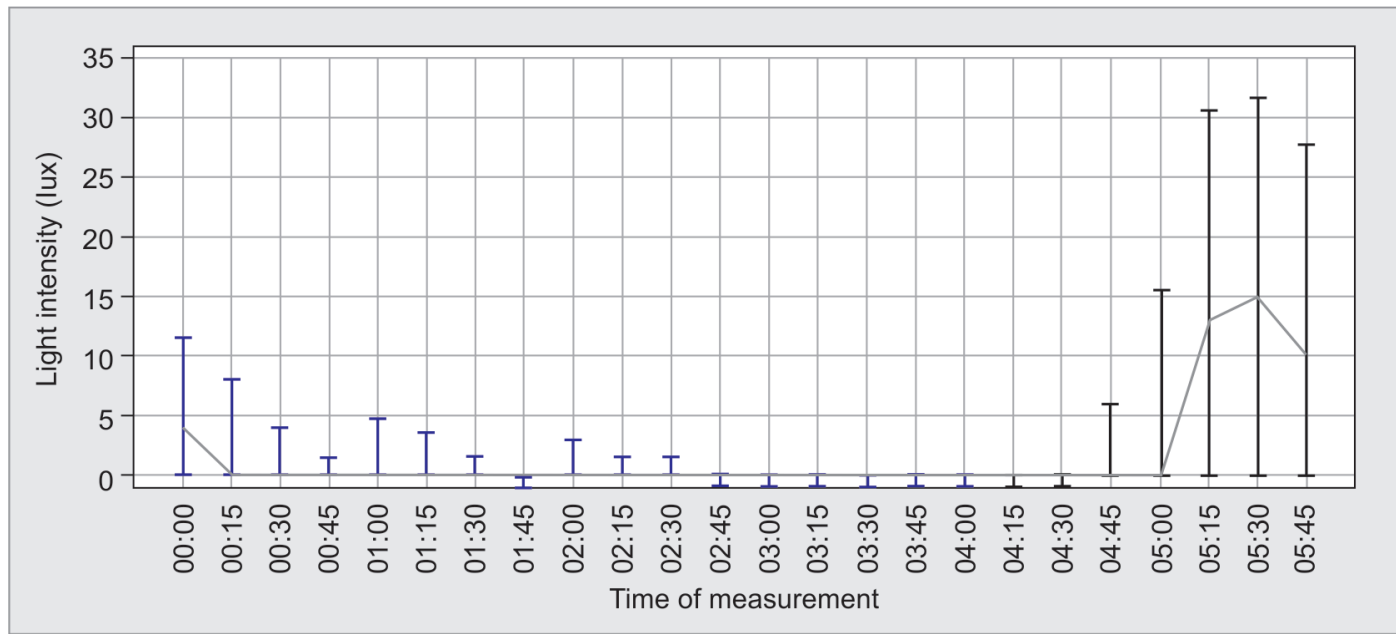

Fig. 2: Light levels at 15 min intervals during sleep-protected time 
Table 2: Correlations between different factors and self-reported quality of sleep in the ICU

\begin{tabular}{lcl}
\hline Factor & Correlation coefficient & Significance level \\
\hline APACHE II & 0.03 & 0.90 \\
SAPS II & -0.03 & 0.88 \\
SOFA & -0.07 & 0.77 \\
$\begin{array}{l}\text { Mean light intensity } \\
\text { (sleep-protected time) }\end{array}$ & 0.33 & 0.13 \\
$\begin{array}{l}\text { Mean light intensity } \\
\text { (before sleep-protected }\end{array}$ & -0.55 & $\mathbf{0 . 0 0 8}$ \\
time) & & \\
$\begin{array}{l}\text { Number of time points } \\
\text { asleep }\end{array}$ & 0.20 & 0.36 \\
$\begin{array}{l}\text { Number of time points } \\
\text { reporting pain }\end{array}$ & 0.21 & 0.34 \\
\hline APACHE Il acute physiogy & & \\
\hline
\end{tabular}

APACHE II, acute physiology and chronic health evaluation II; SAPS II, simplified acute physiology score II; and SOFA, sequential organ failure assessment. Factor that is statistically significant is given in bold

quality of sleep in our study was 49 (IQR 28-71) mm, indicating that the sleep quality was average; however, some authors reported even poorer sleep quality as per RCSQ-34.4 $\pm 5.6 \mathrm{~mm}^{28}$

Light levels during sleep-protected time in our study were low $(11 \pm 9$ lux $)$ and had no impact on sleep duration or sleep quality of patients. This is in concordance with the results of another study reporting light levels of $2.32 \pm 3.65 \mathrm{lux} /$ minute from midnight to $4 \mathrm{am}$ and no impact on circadian rhythm. ${ }^{29}$ Another study that used simulated scenario reported mean levels for minimum lightning of 1-3 lux. ${ }^{30}$

Light levels directly before sleep-protected time in our study were also low ( $13 \pm 5$ lux ) and were correlated with quality of sleep of patients. This result is in line with another study, which showed that high light levels during hours before bedtime can alter circadian rhythm due to delayed melatonin secretion. ${ }^{31}$

\section{Limitations of the Study}

One of the limitations was the number of patients in our study. We included in our study 31 patients. This was partially caused by a population of patients treated in our unit, and many of them were neurological/neurosurgical patients impossible to be included in the study due to neurological status. Another limitation is observational method used to assess duration of sleep in our study (Patient's Sleep Observation Behavioral Tool). Using this tool, we might misjudge patients with eyes closed as asleep; however, as mentioned earlier the use of the reference method (polysomnography) in the ICU is not practical. Lastly, we did not measure sleep during daytime, which might have contributed to the total sleep time of patients; however, it was not practical for the conduct of the study.

\section{ConcLusion}

Mean light levels of $11 \pm 9$ lux during sleep-protected time have no negative impact on quantity and quality of sleep in intensive care unit patients.

\section{References}

1. Wang J, Greenberg H. Sleep and the ICU. Open Crit Care Med J 2013;6(1):80-87. DOI: 10.2174/1874828701306010080.

2. Elliott R, McKinley S, Cistulli P, Fien M. Characterisation of sleep in intensive care using 24-hour polysomnography: an observational study. Crit Care 2013;17(2):R46. DOI: 10.1186/cc12565.
3. Weinhouse GL, Schwab RJ, Watson PL, Patil N, Vaccaro B, Pandharipande $P$, et al. Bench-to-bedside review: delirium in ICU patients: importance of sleep deprivation. Crit Care 2009;13(6):234. DOI: 10.1186/cc8131.

4. Kamdar BB, Niessen T, Colantuoni E, King L, Neufeld KJ, Bienvenu OJ, et al. Delirium transitions in the medical ICU: exploring the role of sleep quality and other factors. Crit Care Med 2014;43:135-141. DOI: 10.1097/CCM.0000000000000610.

5. Tembo AC, Parker V, Higgins I. The experience of sleep deprivation in intensive care patients: findings from a larger hermeneutic phenomenological study. Intensive Crit Care Nurs 2013;29(6):310-316. DOI: 10.1016/j.iccn.2013.05.003.

6. Freedman NS, Gazendam J, Levan L, Pack Al, Schwab RJ. Abnormal sleep/wake cycles and the effect of environmental noise on sleep disruption in the intensive care unit. Am J Respir Crit Care Med 2001;163(2):451-457. DOI: 10.1164/ajrccm.163.2.9912128.

7. Gehlbach BK, Chapotot F, Leproult R, Whitmore H, Poston J, Pohlman $M$, et al. Temporal disorganization of circadian rhythmicity and sleep-wake regulation in mechanically ventilated patients receiving continuous intravenous sedation. Sleep 2012;35(8):1105-1114. DOI: 10.5665/sleep.1998.

8. Pisani MA, Friese RS, Gehlbach BK, Schwab RJ, Weinhouse GL, Jones SF. Sleep in the intensive care unit. Am J Respir Crit Care Med 2015;191(7):731-738. DOI: 10.1164/rccm.201411-2099CI.

9. Gazendam JAC, Van Dongen HPA, Grant DA, Freedman NS, Zwaveling $\mathrm{JH}$, Schwab RJ. Altered circadian rhythmicity in patients in the ICU. Chest 2013;144(2):483-489. DOI: 10.1378/chest.12-2405.

10. Chan MC, Spieth PM, Quinn K, Parotto M, Zhang H, Slutsky AS. Circadian rhythms: from basic mechanisms to the intensive care unit. Crit Care Med 2012;40(1):246-253. DOI: 10.1097/ CCM.0b013e31822f0abe.

11. Carlson D, Chiu W. The absence of circadian cues during recovery from sepsis modifies pituitary-adrenocortical function and impairs survival. Shock 2008;29(1):127-132. DOI: 10.1097/shk.0b013e318142c5a2.

12. Fitzgerald JM, Adamis D, Trzepacz PT, O'Regan N, Timmons S, Dunne C, et al. Delirium: a disturbance of circadian integrity? Med Hypotheses 2013;81(1):568-576. DOI: 10.1016/j.mehy.2013.06.032.

13. Durrington HJ, Clark R, Greer R, Martial FP, Blaikley J, Dark P, et al. 'In a dark place, we find ourselves': light intensity in critical care units. Intensive Care Med Exp 2017;5(1):9. DOI: 10.1186/s40635-017-0122-9.

14. Duffy JF, Czeisler CA. Effect of light on human circadian physiology. Sleep Med Clin 2009;4(2):165-177. DOI: 10.1016/j.jsmc.2009.01.004.

15. Dennis CM, Lee R, Woodard EK, Jeffery S, Catrice W. Benefits of quiet time for neuro-intensive care patients. J Neurosci Nurs 2010;42(4): 217-224. DOI: 10.1097/JNN.0b013e3181e26c20.

16. Patel J, Baldwin J, Bunting P, Laha S. The effect of a multicomponent multidisciplinary bundle of interventions on sleep and delirium in medical and surgical intensive care patients. Anaesthesia 2014;69(6):540-549. DOI: 10.1111/anae.12638.

17. Thompson DR, Hamilton DK, Cadenhead CD, Swoboda S, Schwindel SM, Anderson DC, et al. Guidelines for intensive care unit design. Crit Care Med 2012;40(5):1586-1600. DOI: 10.1097/ CCM.0b013e3182413bb2.

18. Sessler CN, Gosnell MS, Grap MJ, Brophy GM, O'Neal PV, Kimberly A, et al. The Richmond agitation-sedation scale: validity and reliability in adult intensive care unit patients. Am J Respir Crit Care Med 2002;166(10):1338-1344. DOI: 10.1164/rccm.2107138.

19. Vincent JL, Shehabi Y, Walsh TS, Pandharipande PP, Ball JA, Spronk P, et al. Comfort and patient-centred care without excessive sedation: the eCASH concept. Intensive Care Med 2016;42(6):962-971. DOI: 10.1007/s00134-016-4297-4.

20. Kotfis K, Marra A, Ely EW. ICU delirium - a diagnostic and therapeutic challenge in the intensive care unit. Anaesthesiol Intensive Ther 2018;50(2):160-167. DOI: 10.5603/AIT.a2018.0011.

21. Edwards GB, Schuring LM. Pilot study: validating staff nurses' observations of sleep and wake states among critically ill patients, using polysomnography. Am J Crit Care 1993;2(2):125-131. DOI: 10.4037/ajcc1993.2.2.125. 
22. Richards KC, O'Sullivan PS, Phillips RL. Measurement of sleep in critically ill patients. J Nurs Meas 2000;8(2):131-144. DOI: 10.1891/10613749.8.2.131.

23. Ritmala-Castren M, Lakanmaa RL, Virtanen I, Leino-Kilpi H. Evaluating adult patients' sleep: an integrative literature review in critical care. Scand J Caring Sci 2014;28(3):435-448. DOI: 10.1111/scs.12072.

24. Younis MB, Hayajneh F, Batiha AM. Measurement and Nonpharmacologic management of sleep disturbance in the intensive care units: a literature review. Crit Care Nurs Q 2019;42(1): 75-80. DOI: 10.1097/CNQ.0000000000000240.

25. Boyko $\mathrm{Y}$, Ording $\mathrm{H}$, Jennum P. Sleep disturbances in critically ill patients in ICU: how much do we know? Acta Anaesthesiol Scand 2012;56(8):950-958. DOI: 10.1111/j.1399-6576.2012.02672.x.

26. Aitken L, Elliott R, Mitchell M, Davis C, Macfarlane B, Ullman A, et al. Sleep Assessment by patients and nurses in the intensive care: an exploratory descriptive study. Aust Crit Care 2017;30(2):59-66. DOl: 10.1016/j.aucc.2016.04.001.
27. Biren B. Patient nurse interrater reliability and agreement of the Richards-Campbell sleep questionnaire. Am J Crit Care 2012;21(4):261-269. DOI: 10.4037/ajcc2012111.

28. Alsulami G, Rice AM, Kidd L. Prospective repeated assessment of selfreported sleep quality and sleep disruptive factors in the intensive care unit: acceptability of daily assessment of sleep quality. BMJ Open 2019;9(6):e029957. DOI: 10.1136/bmjopen-2019-029957.

29. Verceles AC, Silhan L, Terrin M, et al. Circadian rhythm disruption in severe sepsis: the effect of ambient light on urinary 6-sulfatoxymelatonin secretion. Intensive Care Med 2012;38(5): 804-810. DOI: 10.1007/s00134-012-2494-3.

30. Voigt LP, Reynolds K, Mehryar M, et al. Monitoring sound and light continuously in an intensive care unit patient room: a pilot study. J Crit Care 2017;39:36-39. DOI: 10.1016/j.jcrc.2016.12.020.

31. Burgess $\mathrm{HJ}$, Molina TA. Home lighting before usual bedtime impacts circadian timing: a field study. Photochem Photobiol 2014;90(3): 723-726. DOI: 10.1111/php.12241. 